\title{
Epitranscriptomic profile of Lactobacillus agilis and its adaptation to growth on inulin
}

\author{
Hongzhou Wang ${ }^{1 \dagger}$, Jennifer H. Simpson ${ }^{1 \dagger}{ }^{\text {, Madison E. Kotra }}{ }^{1}$, Yuanting Zhu², Saumya Wickramasinghe², \\ David A. Mills ${ }^{2}$ and Norman H. L. Chiu ${ }^{1,3^{*}}$ (D)
}

\begin{abstract}
Objective: Ribonucleic acids (RNA) are involved in many cellular functions. In general, RNA is made up by only four different ribonucleotides. The modifications of RNA (epitranscriptome) can greatly enhance the structural diversity of RNA, which in turn support some of the RNA functions. To determine whether the epitranscriptome of a specific probiotic is associated with its adaptation to the source of energy, Lactobacillus agilis (YZ050) was selected as a model and its epitranscriptome was profiled and compared by using mass spectrometry.

Results: The L. agilis epitranscriptome (minus rRNA modifications) consists of 17 different RNA modifications. By capturing the L. agilis cells during exponential growth, reproducible profiling was achieved. In a comparative study, the standard source of energy (glucose) in the medium was substituted by a prebiotic inulin, and a downward trend in the L. agilis epitranscriptome was detected. This marks the first report on a system-wide variation of a bacterial epitranscriptome that resulted from adapting to an alternative energy source. No correlation was found between the down-regulated RNA modifications and the expression level of corresponding writer genes. Whereas, the expression level of a specific exonuclease gene, RNase J1, was detected to be higher in cells grown on inulin.
\end{abstract}

Keyword: RNA modifications, Epitranscriptome, Lactobacillus agilis, Inulin

\section{Introduction}

Collectively, all the RNA molecules in a specific group of cells are referred as a transcriptome. In order to achieve some of the RNA functionalities, the RNA structure can be altered by more than 170 different RNA modifications $[1,2]$. The presence of a RNA modification is the result of an enzymatic reaction of its corresponding writer enzyme. In contrast, RNA modification can be removed by a different enzyme called eraser. To recognize the importance of RNA modifications to the RNA structures and functions, the term of epitranscriptome was coined by Mason and his associates [3]. There are reports

\footnotetext{
*Correspondence: prof.chiu@gmail.com

${ }^{\dagger}$ Hongzhou Wang and Jennifer H. Simpson both authors have equal contributions

${ }^{1}$ Department of Chemistry and Biochemistry, University of North Carolina Greensboro, Greensboro, NC, USA

Full list of author information is available at the end of the article
}

indicating specific epitranscriptomes are linked to a variety of health-related issues $[4,5]$. With the interests in studying epitranscriptomes, a number of methods for analyzing RNA modifications have been developed [6,7]. Among those methods, mass spectrometric (MS) based method is the only universal approach for detecting different RNA modifications.

Lactobacillus species are common constituents of gastrointestinal tracts [8], and have been used as probiotics [9]. Prebiotics are defined as substrates that are utilized by microorganisms conferring health benefits [10]. One of the most commonly used prebiotics is inulin [11]. Since inulin cannot be metabolized by human digestive enzymes, the digestion of inulin relies on gut microbes [12]. In this report, we use the MS method to profile the $L$. agilis epitranscriptome, and subsequently determine whether the $L$. agilis epitranscriptome is involved in the adaptation to inulin.

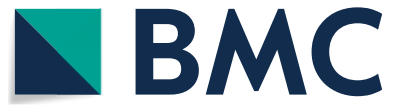

(c) The Author(s) 2021. This article is licensed under a Creative Commons Attribution 4.0 International License, which permits use, sharing, adaptation, distribution and reproduction in any medium or format, as long as you give appropriate credit to the original author(s) and the source, provide a link to the Creative Commons licence, and indicate if changes were made. The images or other third party material in this article are included in the article's Creative Commons licence, unless indicated otherwise in a credit line to the material. If material is not included in the article's Creative Commons licence and your intended use is not permitted by statutory regulation or exceeds the permitted use, you will need to obtain permission directly from the copyright holder. To view a copy of this licence, visit http://creativecommons.org/licenses/by/4.0/. The Creative Commons Public Domain Dedication waiver (http://creativecommons.org/publicdomain/zero/1.0/) applies to the data made available in this article, unless otherwise stated in a credit line to the data. 


\section{Main text Methods}

E. coli alkaline phosphatase, Benzonase nuclease and bovine serum albumin (BSA) were purchased from Sigma-Aldrich (St. Louis, MO, USA). The venom exonuclease phosphodiesterase I was purchased from Worthington Biochemical Corp. (Lakewood, NJ, USA). All other solvents were purchased from Thermo Fisher Scientific (Waltham, MA).

\section{Culturing of L. agilis}

A Lactobacillus agilis strain named YZ050 was previously isolated from dairy cow fecal samples in our lab and showed the capability to ferment inulin [13]. The stock was streaked on MRS plates. After $24 \mathrm{~h}$, a MRS broth was inoculated and cultivated at $37{ }^{\circ} \mathrm{C}$ under anaerobic conditions. The overnight culture (1\%) was inoculated into basal MRS media supplemented with $1 \%$ inulin or $1 \%$ glucose. After $\sim 5 \mathrm{~h}$, samples were taken for RNA extraction at mid-log phase.

\section{Extraction of RNA}

Cells were pelleted and resuspended in $1 \mathrm{~mL}$ RNAprotect Bacteria Reagent (Qiagen Inc, Valencia). The cells were washed twice with $1 \mathrm{X}$ PBS and pre-lysed with $250 \mu \mathrm{L}$ $50 \mathrm{~g} / \mathrm{L}$ lysozyme and $120 \mu \mathrm{L} 1000$ units/mL mutanolysin. Total RNA was extracted using the RNeasy mini kit (Qiagen Inc, Valencia). Total RNA samples were DNasetreated twice, and the absence of genomic DNA was confirmed by PCR.

\section{Depletion of ribosomal RNA (rRNA)}

Ribosomal RNA was removed using the RiboMinus Transcriptome Isolation Kit, (Thermo Fisher, Waltham, MA). The integrity of RNA was assayed using the 2100 Bioanalyzer (Agilent Technologies, Santa Clara, CA).

\section{Digestion of rRNA-depleted RNA}

Each RNA sample was digested in an enzymatic reaction of $25 \mu \mathrm{L}$ at $37^{\circ} \mathrm{C}$ for $3 \mathrm{~h}$, which contained $5 \mu \mathrm{g}$ rRNAdepleted RNA, 0.05 units phosphodiesterase I, 0.5 units alkaline phosphatase, 5 units benzonase, $50 \mathrm{mM}$ Tris$\mathrm{HCl}(\mathrm{pH} 8.0), 1 \mathrm{mM} \mathrm{MgCl}$ and $0.1 \mathrm{mg} / \mathrm{mL}$ BSA [14]. After removing the enzymes with $3 \mathrm{~K}$ MWCO spin filter at $14,000 \mathrm{~g}$ for $15 \mathrm{~min}$ (Pall Corporation, Port Washington, NY), the digested RNA sample was diluted in deionized water to $50 \mathrm{ng} / \mu \mathrm{L}$.

\section{UPLC-MS/MS analysis of digested RNA}

An Acquity ultra-high performance liquid chromatography (UPLC) system (Waters Corporation, Milford, MA) which was equipped with an Acquity HSS T3 column $(2.1 \times 50 \mathrm{~mm}, 1.8 \mu \mathrm{m})$ and a HSS T3 VanGuard pre-column $(2.1 \times 5 \mathrm{~mm}, 1.8 \mu \mathrm{m})$ at $30{ }^{\circ} \mathrm{C}$ was used. After injecting $10 \mu \mathrm{L}$ of sample, the elution was carried out with a binary solvent system, in which solvent A contained water and $0.01 \%(\mathrm{v} / \mathrm{v})$ formic acid, and solvent B contained $50 \%$ acetonitrile and $0.01 \%(\mathrm{v} / \mathrm{v})$ formic acid at a flowrate of $0.4 \mathrm{~mL} / \mathrm{min}$. The gradient elution profile initiated at 100:0 (A:B) from 0.0 to $0.5 \mathrm{~min}$., ramping to $70: 30$ from 0.5 to $9 \mathrm{~min}$, followed by 50:50 from 9 to $10 \mathrm{~min}$, and ended with 0:100 from 10 to $17 \mathrm{~min}$. Randomized injections were used. The negative control was prepared without any RNA sample.

Tandem mass spectrometry (MS/MS) was performed on a Q Exactive Plus (Thermo Fisher Scientific, Waltham, MA) in the positive mode with ESI at $425^{\circ} \mathrm{C}$ and $3.5 \mathrm{kV}$. Sheath and auxiliary gas flow were at 50 and 13 arbitrary units, respectively. Data was acquired with an inclusion list of calculated $\mathrm{m} / \mathrm{z}$ of all known RNA modifications. The mass calibration was performed using a canonical ribonucleoside standard mixture $(3 \mathrm{ng} / \mu \mathrm{L})$. Data analysis was carried out with Xcalibur (Thermo Fisher Scientific, Waltham, MA) restricting the precursor ion to $\leq 5 \mathrm{ppm}$ accuracy and its retention time to $\leq 0.1 \mathrm{~min}$.

\section{RNA sequencing (RNAseq)}

Each library was generated from 20 ng rRNA-depleted RNA sample using the Kapa Hyper Stranded RNA-seq kit (KapaBiosystems, Cape Town, South Africa). The consistency of the libraries was verified by 2100 Bioanalyzer. The libraries were quantified by fluorometry and sequenced on a NextSeq 500 (Illumina, San Diego, CA) with paired-end $75 \mathrm{p}$ reads. The sequence files were processed using the CLC-Bio Genomics Workbench (CLC Bio, Denmark).

\section{Results and discussion}

\section{Analysis of L. agilis epitranscriptome}

The notions for epitranscriptome to be a standalone investigation include a single RNA modification can potentially alter the RNA interactions [15]. There are also evidence showing unique epitranscriptomes are associated with specific phenotypes [16]. Together with the discovery of various writer genes for RNA modifications, a specific epitranscriptome is considered to represent a set of specific codes for regulating cellular activities [17]. Our initial efforts focused on establishing the profile of L. agilis epitranscriptome. Among various types of RNA, ribosomal RNA (rRNA) makes up $\sim 80 \%$ of total RNA [18]. To better witness bacterial gene expression, rRNA is often depleted from the RNA samples prior to sequencing. Equivalently, rRNA was also removed in our protocol, otherwise would reduce the detectability of RNA modifications that are unique in other types of RNA. The removal of rRNA can also enhance our ability to 
detect any variations on the levels of some specific RNA modifications.

The results obtained from analyzing all the detectable ribonucleosides in a $L$. agilis sample with a signalto-noise ratio of $\geq 2$ are shown in Table 1 . To ensure the low abundant RNA modifications could be detected, the chromatography and signal intensity in the UPLC-MS/ MS analysis were optimized. As low as $0.4 \mathrm{pg} / \mu \mathrm{L}$ of each canonical ribonucleoside standard were detected in our calibration experiments. For identifying the RNA modification, both MS and MS/MS data must match with the expected values with $<5 \mathrm{ppm}$ error. For the MS/MS measurements, at least two fragment ions were identifiable. The profiling was repeated four times with different samples, and the same profile of RNA modifications were detected each time. To the best of our knowledge, this is the first time the profile of $L$. agilis epitranscriptome (minus the rRNA modifications) is reported.

Before determining whether there were any variations on the level of each specific RNA modification, the use of our method to perform accurate quantitative analysis was evaluated. Specifically, a calibration experiment with a series of standard dilutions was performed. The results indicate the linearity and the dynamic range of the four canonical ribonucleoside standards match or exceed the earlier reports with $<6 \%$ relative standard deviation $(\mathrm{n}=3)[19,20]$.

As shown by the error bars in Fig. 1, there was no significant variation on the level of each RNA modification among the four repeated profiling of glucose-associated $L$. agilis epitranscriptome. This indicates the L. agilis epitranscriptome reaches an equilibrium state when the cells were harvested during the exponential growth.

\section{Glucose-associated vs. inulin-associated L. agilis epitranscriptome}

The reproducibility of the $L$. agilis epitranscriptomic profile prompted us to investigate whether the $L$. agilis epitranscriptome would become different when different prebiotic was used. As shown in Fig. 1, the RNA modifications found in the inulin-associated $L$. agilis epitranscriptome match with those listed in Table 1 . However, there is an obvious downward trend when the cells were cultivated in inulin instead of glucose. However, the fold change of each individual RNA modification was not uniform, with 2'-O-methyladenosine (Am)

Table 1 LC-MS data obtained from the glucose-associated L. agilis transcriptome in the absence of rRNA

\begin{tabular}{|c|c|c|c|}
\hline Ribonucleoside detected and its short name & Retention time $^{\mathrm{a}}(\mathrm{min})$ & Measured mass $^{\mathbf{b}}(\mathrm{Da})$ & $\begin{array}{l}\text { Mass } \\
\text { accuracyc } \\
(p p m)\end{array}$ \\
\hline Cytidine, C & 0.90 & 244.0935 & 0.7 \\
\hline Dihydrouridine, D & 0.94 & 247.0933 & 3.3 \\
\hline Pseudouridine, $Y$ & 0.98 & 245.0775 & 3.0 \\
\hline 1-Methyladenosine, m1A & 1.55 & 282.1205 & 3.1 \\
\hline 5-Methylcytidine, m5C & 1.63 & 258.1093 & 3.2 \\
\hline Uridine, U & 2.00 & 245.0776 & 3.3 \\
\hline 7-Methylguanosine, m7G & 2.54 & 298.1155 & 3.1 \\
\hline 2'-O-Methylcytidine, Cm & 2.79 & 258.1093 & 3.2 \\
\hline 2'-O-Methylpseudouridine, Ym & 2.99 & 259.0934 & 3.5 \\
\hline Guanosine, G & 3.99 & 284.0997 & 2.8 \\
\hline 5-Methyluridine, m5U & 4.06 & 259.0933 & 3.2 \\
\hline Adenosine, A & 4.45 & 268.1048 & 2.8 \\
\hline 3-Methyluridine, m3U & 4.86 & 259.0933 & 3.1 \\
\hline 1-Methylguanosine, m1G & 5.27 & 298.1155 & 3.2 \\
\hline 2'-O-Methylguanosine, Gm & 5.27 & 298.1155 & 3.2 \\
\hline N2-methylguanosine, m2G & 5.53 & 298.1155 & 3.2 \\
\hline N4-acetylcytidine, ac4C & 5.61 & 286.1034 & 0.2 \\
\hline 2'-O-Methyladenosine, Am & 5.64 & 282.1204 & 2.8 \\
\hline N6-methyladenosine, m6A & 6.31 & 282.1206 & 3.2 \\
\hline N6,N6-dimethyladenosine, m6,6A & 7.93 & 296.1354 & 0.2 \\
\hline N6-threonylcarbamoyladenosine, t6A & 8.04 & 413.1417 & 0.8 \\
\hline
\end{tabular}

\footnotetext{
${ }^{a} \pm 0.01 \mathrm{~min}$

${ }^{\mathrm{b}}$ Mass of protonated precursor ion

${ }^{c}$ Reference to the monoisotopic mass of protonated precursor ion
} 


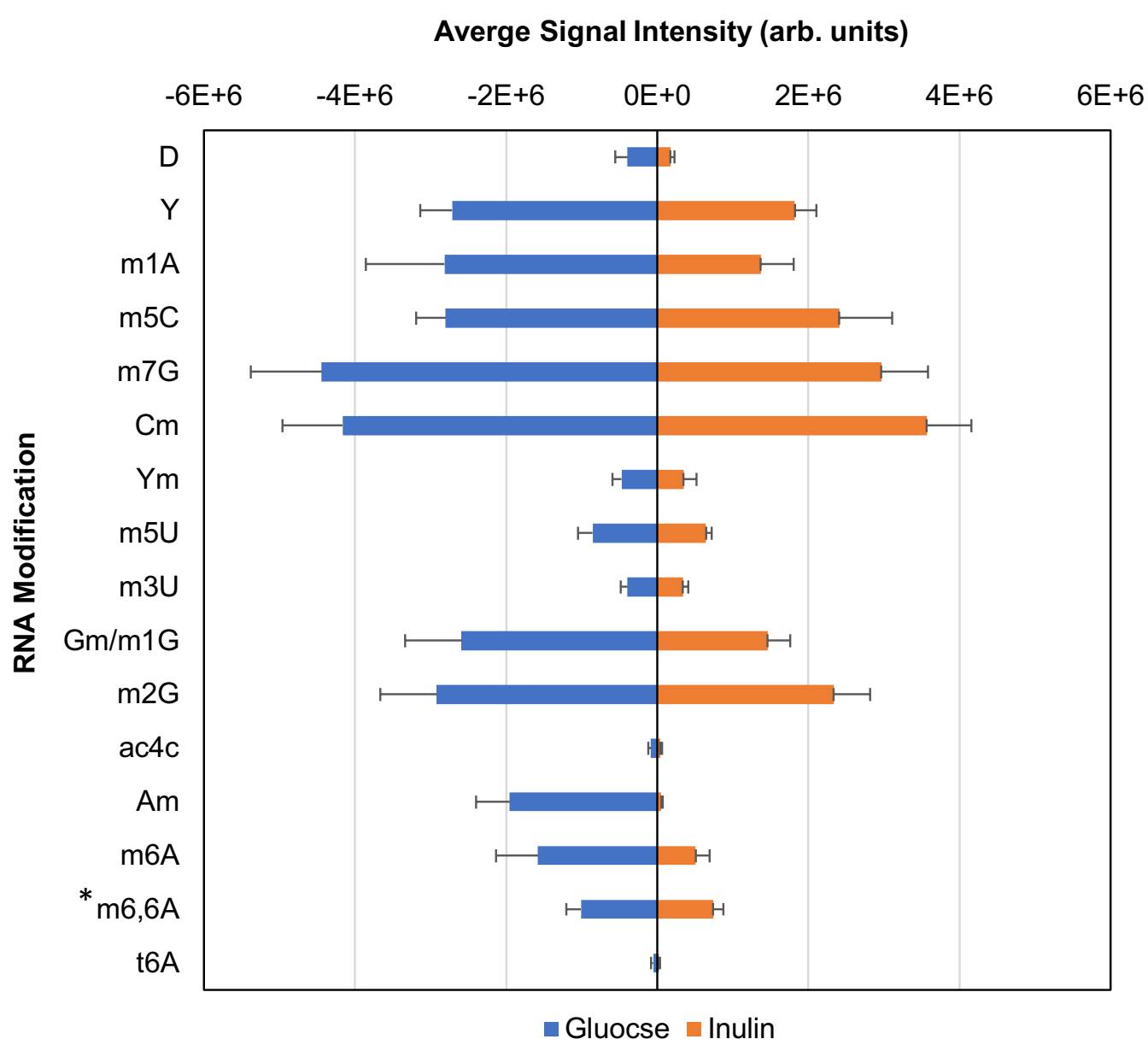

Fig. 1 Mirrored histogram of glucose- or inulin-associated L. agilis epitranscriptomic profiles obtained from rRNA-depleted total RNA. ${ }^{*}$ The signals of m6,6A were scaled down 10-folds. Each error bar represents one standard deviation with $n \leq 12$

to be down regulated most. From the chemical point of view, the $2^{\prime}$-O-methylation can disrupt the interactions between $2^{\prime}$-O-methylated RNA and RNase, thus protecting the $2^{\prime}$-O-methylated RNA from the RNase activity [21]. Therefore, when the level of Am was lowered in the inulin-associated $L$. agilis transcriptome, it would allow the $L$. agilis transcriptome to be turned over more effectively via the RNase digestion, which could be one way to rearrange the composition of the L. agilis transcriptome.

Among all seventeen RNA modifications witnessed in the L. agilis epitranscriptome, six of them were down regulated more than the average fold change of 0.65 . The top six down-regulated RNA modifications include dihydrouridine (D), 1-methyladenosine (m1A), 4-acetylcytidine (ac4C), 2'-O-methyladenosine (Am), N6-methyladenosine (m6A) and N6-threonylcarbamoyl-adenosine (t6A). In the case of D modification, the hydrogenation at the 5 and 6 positions of uridine eliminate the only $\pi$ bonding, thus weakening the effects of base stacking [22].
Whereas, the modifications of $\mathrm{m} 1 \mathrm{~A}$, ac4C, m6A and t6A would interfere with the Watson-Crick base pairing. Therefore, the down regulation of those modifications could potentially change some of the RNA folding and/ or annealing.

To investigate the underlying reason for the down regulation of $L$. agilis epitranscriptome, we performed an untargeted gene expression analysis and compared the expression levels in the cell cultures. First, we focused on the writer and eraser genes that correspond to the top six down-regulated RNA modifications (Fig. 2). However, the DNA sequence of those eraser genes are not known. Thus, our data analysis was limited to the writer genes. The results showed that there was no difference on the expression levels of the writer for t6A, D, m1A and ac4C modifications. The writer gene for Am in L. agilis is not known; and no transcript corresponding to the m6A writer gene could be detected. An alternative mechanism to lower 


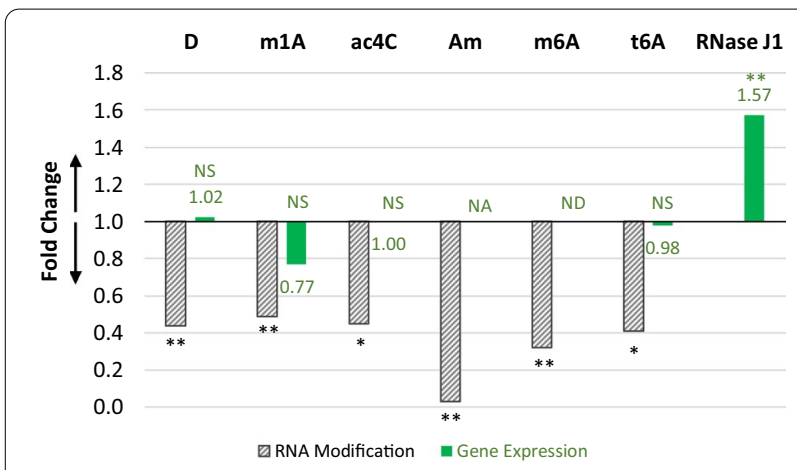

Fig. 2 Fold change on the top six down-regulated RNA modifications and the expression level of their corresponding writer gene or RNase $\mathrm{J} 1$ that resulted from switching glucose to inulin in the cultivating L. agilis. The numerical numbers show the exact fold changes in the expression level. NS not significant, NA gene identity not available, ND transcript not detected; ${ }^{*} p \leq 0.05,{ }^{* *} p \leq 0.01$

the level of specific RNA modifications could be due to an increased cellular activity on degrading the modified RNA molecules. For this reason, the expression levels of all detectable ribonuclease in $L$. agilis were compared, which included RNase 3, RNase HI, RNase HII, J1, RNase R, RNase Y and RNase Z. In the case of RNase J1, a significant increase on its expression level was found among the inulin samples (Fig. 2). Whereas, no differences were detected for all the other RNases. Hence, based on the scope of this study, we speculate the down regulation of the inulin-associated $L$. agilis epitranscriptome could be linked to the higher expression level of RNase J1 when inulin was used instead of glucose to cultivate the cells.

\section{Conclusion}

When $L$. agilis cells were cultivated with glucose being the sole source of energy, the L. agilis epitranscriptome consists of seventeen different RNA modifications at variable abundancy. There was a downward trend across the entire L. agilis epitranscriptome when the cells were exposed to inulin instead of glucose. To the best of our knowledge, this marks the first report on a system-wide variation of a bacterial epitranscriptome that resulted from adapting to an alternative source of energy. Based on our comparative study on gene expression, the down-regulated inulin-associated L. agilis epitranscriptome could be linked to an elevated RNase J1 activity. Overall, these results further strengthen the association of a unique epitranscriptome to a specific cellular activity [23].

\section{Limitations}

Although all the above observations were limited to the selected strain of L. agilis, the experimental approach is applicable to study the other strains. For the MS analysis, due to the lack of available standards, the identification of each RNA modification was limited to the intrinsic accuracy from using high resolution mass spectrometry. Furthermore, the RNA modifications below the limit of detection would not be detectable and missed out from the reported profile.

\section{Abbreviations \\ ac4C: 4-Acetylcytidine; Am: 2'-O-Methyladenosine; D: Dihydrouridine; PCR: Polymerase chain reaction; L. agilis: Lactobacillus agilis; M1A: 1-Methyladeno- sine; m6A: N6-Methyladenosine; MS: Mass spectrometry; RNA: Ribonucleic acid; RNase: Ribonuclease; rRNA: Ribosomal ribonucleic acid; t6A: N6-Threonyl- carbamoyl-adenosine; UPLC-MS/MS: Ultra-high performance liquid chroma- tography tandem mass spectrometry.}

\section{Acknowledgements}

The authors wish to acknowledge the supports from UNCG and thank Lily Barrett for her assistance. A special thanks to Prof. Carlito Lebrilla for his helpful discussion and Daniel Todd for his technical support.

\section{Authors' contributions}

DM and NC: Conceived, designed, supervised the project and wrote the manuscript. HW, JS, MK, YZ and SW: Performed the experiments and analyzed the data. All authors read and approved the final manuscript.

\section{Funding}

YZ was funded via a China Scholarship Council (CSC) and DM acknowledges funding from the Peter J. Shields Endowed Chair for Dairy Food Science.

\section{Availability of data and materials}

The datasets used and/or analyzed during the current study are available from the corresponding author on reasonable request.

\section{Declarations}

Ethics approval and consent to participants

No human participants, human material, or human data were involved in this study.

\section{Consent for publication}

Not applicable.

\section{Competing interests}

The authors have no conflict of interests.

\section{Author details}

${ }^{1}$ Department of Chemistry and Biochemistry, University of North Carolina Greensboro, Greensboro, NC, USA. ${ }^{2}$ Department of Food Science and Technology, University of California, Davis, CA, USA. ${ }^{3}$ Joint School of Nanoscience and Nanoengineering, University of North Carolina Greensboro, Greensboro, NC, USA.

Received: 9 January 2021 Accepted: 9 April 2021

Published online: 21 April 2021

\section{References}

1. Boccaletto P, et al. MODOMICS: a database of RNA modification pathways. 2017 update. Nucleic Acids Res. 2018;46(D1):D303-7. 
2. Ontiveros RJ, Stoute J, Liu KF. The chemical diversity of RNA modifications. Biochem J. 2019;476(8):1227-45.

3. Saletore Y, Meyer K, Korlach J, Vilfan ID, Jaffrey S, Mason CE. The birth of the epitranscriptome: deciphering the function of RNA modifications. Genome Biol. 2012;13(10):175-86.

4. Yanas A, Liu KF. RNA modifications and the link to human disease. Methods Enzymol. 2019;626:133-46.

5. Barbieri I, Kouzarides T. Role of RNA modifications in cancer. Nat Rev Cancer. 2020;20(6):303-22.

6. Chen B, Yuan B, Feng Y. Analytical methods for deciphering RNA modifications. Anal Chem. 2019;91:743-56.

7. Lauman R, Garcia BA. Unraveling the RNA modification code with mass spectrometry. Mole Omics. 2020. https://doi.org/10.1039/C8MO00247A.

8. Heeney DD, Gareau MG, Marco ML. Intestinal Lactobacillus in health and disease, a driver or just along for the ride? Curr Opin Biotech. 2018:49:140-7.

9. Lebeer $\mathrm{S}$, et al. Identification of probiotic effector molecules: present state and future perspectives. Curr Opin Biotech. 2018;49:217-23.

10. Gibson GR, et al. Expert consensus document: the International Scientific Association for Probiotics and Prebiotics (ISAPP) consensus statement on the definition and scope of prebiotics. Nat Rev Gastroenterol. 2017;14:491-502.

11. Barclay T, et al. Inulin — a versatile polysaccharide with multiple pharmaceutical and food chemical uses. J Excip Food Chem. 2010;1(3):27-50.

12. Le Bastard Q, Chapelet G, Javaudin F, Lepelletier D, Batard E, Montassier E. The effects of inulin on gut microbial composition: a systematic review of evidence from human studies. Eur J Clin Microbiol Infect Dis. 2020;39(3):403-13.

13. Zhu Y, Liu J, Lopez JM, Mills DA. Inulin fermentation by Lactobacilli and Bifidobacteria from dairy calves. Appl Environ Microbiol. 2020. https://doi. org/10.1128/AEM.01738-20.
14. Su D, et al. Quantitative analysis of ribonucleoside modifications in tRNA by HPLC-coupled mass spectrometry. Nat Protoc. 2014;9(4):828-41.

15. Lewis CJ, Pan T, Kalsotra A. RNA modifications and structures cooperate to guide RNA-protein interactions. Nat Rev Mol Cell Biol. 2017;18(3):202-10.

16. Ranjan N, Leidel SA. The epitranscriptome in translation regulation: $m R N A$ and tRNA modifications as the two sides of the same coin? FEBS Lett. 2019;593(13):1483-93.

17. Kadumuri RV, Janga SC. Epitranscriptomic code and its alterations in human disease. Trends Mol Med. 2018;24(10):886-903.

18. Rosenow C, et al. Prokaryotic RNA preparation methods useful for high density array analysis: comparison of two approaches. Nucleic Acids Res. 2001;29(22):e112.

19. Basanta-Sanchez M, et al. Attomole quantification and global profile of RNA modifications: epitranscriptome of human neural stem cells. Nucleic Acids Res. 2016:44:1-10.

20. He $L$, et al. Simultaneous quantification of nucleosides and nucleotides from biological samples. J Am Soc Mass Spectrom. 2019;30:987-1000.

21. Egli $M$, et al. Probing the influence of stereoelectronic effects on the biophysical properties of oligonucleotides: comprehensive analysis of the RNA affinity, nuclease resistance, and crystal structure of ten 2'-O-ribonucleic acid modifications. Biochemistry. 2005;44:9045-57.

22. Dalluge JJ, et al. Posttranscriptional modification of tRNA in psychrophilic bacteria. J Bacteriol. 1997;179(6):1918-23.

23. Huber SM, Leonardi A, Dedon PC, Begley TJ. The versatile roles of the tRNA epitranscriptome during cellular reponses to toxic exposures and environmental stree. Toxics. 2019;7:17-35.

\section{Publisher's Note}

Springer Nature remains neutral with regard to jurisdictional claims in published maps and institutional affiliations.
Ready to submit your research? Choose BMC and benefit from:

- fast, convenient online submission

- thorough peer review by experienced researchers in your field

- rapid publication on acceptance

- support for research data, including large and complex data types

- gold Open Access which fosters wider collaboration and increased citations

- maximum visibility for your research: over $100 \mathrm{M}$ website views per year

At BMC, research is always in progress.

Learn more biomedcentral.com/submissions 